\title{
CONTRIBUTION \\ A L'HISTOIRE DE LA HOUILLE BLANCHE ET LA PART DE LA SAVOIE. DANS LA CONQUÊTE DE L'ÉNERGIE HYDROÉLECTRIQUE
}

\author{
Victor SYLVESTRE \\ Ingénieur A. M. et I.E.G. \\ Lauréat de la Houlle Blanche de IJIniversité de Grenoble \\ Médaille d'Or à l'Exposition Internationale de la Houille Blanche de Grenoble 1725
}

\section{LA FORCE DES EAUX DANS L'ANTIQUITÉ}

Dès la plus haute aniquité, les hommes utilisèrent la force des eaux pour faire mouvoir des artifices et si l'on en croit l'histoire, dès 135 avant Jésus-Christ, Ctésibius inventa une roue a augets.

La roue du moulin naîi, dans le bassin oriental de la Méditerranée, dès le début de l'ère chrétienne et à cette époque, à Constantinople, les moulins à blé jusqualors actionnés à bras par les esclaves, les servantes chantent «Dameter", la déerse des eaux qui vient soulager leur peine.

Le moulin à eau est une conquète du basEmpire. C'est lui que trouva le moyen àge dans la succession de l'empire romain. Le regrette Mare BLoch, de la Sorbonne, en a montré très in elligemment son aire d'expansion, prouvant qu'il fut le moteur le plus habituel, avani l'introduction du moulin à vent, resté inconnu de l'Italie et de l'Afrique méditerranéenne.

Mais si l'on savait par les textes que le moalin à eau était connu de l'antiquité et entra dans le domaine de l'utilisation pratique avec le basEmpire, aucun monument de ce genre n'avait encore été identifié.

Ce n'est que depuis une dizaine d'années a peine que l'hydraulique romaine vient d'être l'objet de découvertes les plus intéressantes, en trois points de l'Empire, comme l'a signalé M. BENoIT, en 1939. Ce sont :

- A Venafro, dans la province de Naples, reconstituition d'une roue pendante sur rivière, d'ap'rès les empreintes calcaires laissées par la roue à aubes d'un moulin.

- A Athènes, dans les fouilles de l'Agora, dégagement d'un moulin à grains, avec bief, actionné par une chute d'eau, et chambre de meunerie, datant du $\mathrm{v}^{0}$ siècle après notre ère.

- A Arles, découverte dune vériable usine de meunerie actionnée par un aqueduc et remontant aux réformes du régime fiseal de l'Annone, sous Dioclétien et Constantin (260-300).

L'établissement hydraulique d'Arles recevait sa force motrice d'un aqueduc long d'une dizaine de kilomètres, sorte de canal d'amenée aboutissant sur une crête rocheuse formant à l'arrivée une chute de dix-huit mètres. C'était sans doute, à l'époque, la plus haute chute dis monde! Deux canaux parallèles utilisaient chacun cette dénivellation en sept ou huit chutes, formant autant de biefs de moulin, auxquels correspondaient autant de chambres de meunerie.

Ainsi par ce plan ingénieux, l'ingénieur de cette première chute d'Arles avait rassemble dans un espace restreint de 61 mètres de Iongueur sur 20 de largeur, une véritable usine qui groupait, sans doute, seize chambres de meune'rie, disposées de part et d'autre d'un escalier central, véritable hall servant d'accès et de dégagement. Voilà pour la partie technique que cette découverie apporte à la connaissance de la science industrielle des anciens, mais comme l'a montré M. Brovort, l'usine arlésienne 


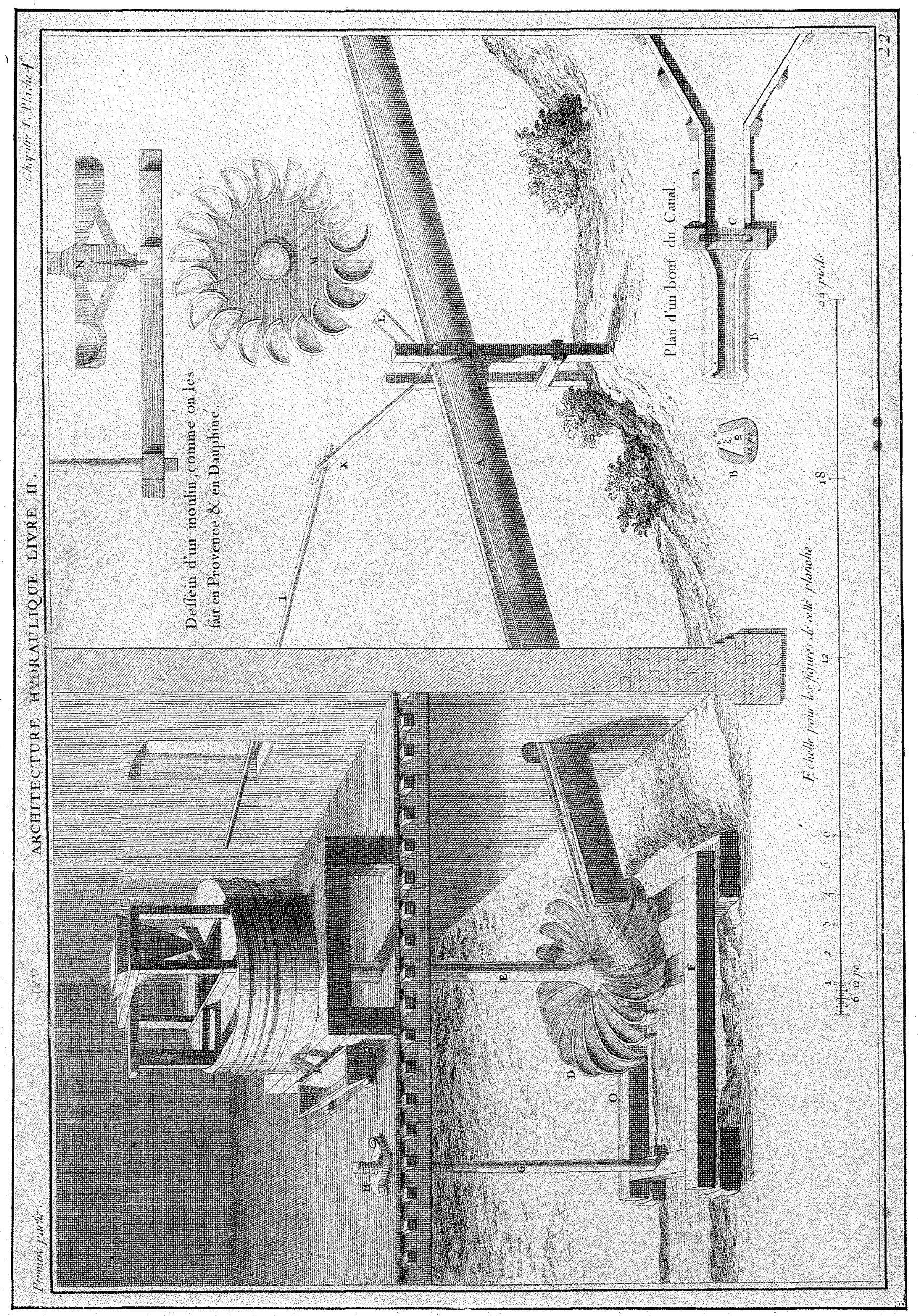


par son ampleur est un des documents les plus intéressants sur les transformations de la vie économique sous le bas-Empire.

Construite à une dizaine de kilomètres d'Arles, au point où la dénivellation abrupte du terrain permettait de capter naturellement une chute d'eau, elle avait donc entrainé la création d'un véritable centre industriel suburbain et donné naissance à tout le mécanisme compliqué inhérent à la grande industrie, avec sa comptabilité, ses règlements du travail et l'organisation délicate de l'approvisionnement des matières; premières et de la distribution ou de la vente des produits manufacturés.

Cette usine était, à n'en pas douter, une fabrique d'Etal, et son importance même, esí bien disne d'illustrer la nature de la révolution sociale que marquèrent les réformes de l'Annone, sous Dioclétien et Constantin : à la liberté du travail familial et artisanal du haut-Empire succède un socialisme d'Etat. A l'atelier du houlanger, Ia pistrina, oì l'artican moud son grain et le transforme en pain, se substitue une meunerie spécialisée, distincte de la boulangerie. Nous assistons là, dans cette première chute utilisée en France, à la naissance de la grande industrie d'Etat.

Ainsi, comme l'a bien vu M. BExort, la meunerie hydraulique d'Arles, cet ancêtre de l'usine, constitue le précieux témoin d'une tentative éphémère de transformation des méthodes de production à la fin de l'empire romain. Retenons qu'elle constitue surtout pour nous hydrauliciens, le premier équipement sur le sol de France, d'une chute d'eat subdivisée, pour des raisons lechniques de la roue à aubes, en deux séries paralleles de huit pelites chutes actionnant chacune une roue de moulin.

Une histoire du moulin à eau dans les régions du midi, en Provence comme dans le sud-est, montrerait que c'est pour lui que furent ouverts tous les canaux qui servent aujourd'hui à l'irrigation.

Dès 1101, la concession du canal de Vaucluse au chapitre d'Avignon permit à ce dernie? d'équiper plusieurs moulins à blé et à foulon : ce sera l'origine du fameux canal de la Duransole, concédé en 1229 par les Consuls, dans un but exclusif de la force hydraulique et qui ne servira que vers la fin du xv" siècle à l'irrigation de la campagne avignonnaise, dont il a fait la fortune.

Dans la région d'Árles, les eaux de la Dirance de Saint-Gabriel, sans doute canalisées dès l'époque romaine, actionnaient au $\mathrm{xi}^{\mathrm{e}}$ siècle les moulins de Saint-Gabriel.

Le. $1 . ., x, y, \ldots, \ldots$ gués par l'archevêque vers $1162-80$, montrent que le moulin a vent, qui paraît pour la premiere fois en Provence, est un mode de mouture peu familier, dont le prix est plus élevé que celui du moulin à eau; le droit de mouture de celui-ci (molendinum aque) était en effet du $1 / 30^{\circ}$, tandis qu'il était du $1 / 20$ e dans le moulin à vent (molendinum de aure).

BELmor, dans son célèbre traité d'hydraulique (1737), donne une description détaillée des importants moulins du Basacle, à Toulouse, dans lesquels, vingt-cing rouets actionnaient chacun une meule à axe vertical, véritable usine hydraulique, dont les rouets par leur disposition en pales d'hélice alimentés par tourbillon, se rapprochent étrangement de nos turbines les plus modernes, Ies turbines à hélices pour basses chutes du type Kaplan.

Cette ressemblance technique a paru tellement surprenante aux ingénieurs du $\mathrm{xx}^{*}$ siècle, que notre regretté compatriote l'ingénieur-hydraulicien Georges Rours, avait reproduit et fait fonctionner un modèle réduit d'un rouet des moulins du Basacle à l'exposition rétrospective organisée par la Chambre Syndicale des Forces Hydrauliques, en 1925, à l'Exposition de la Houile Blanche, à Grenoble, et cela pou'r bien montrer au monde, combien les racines de l'utilisation des forces hydrauliques étaient ancrées en France.

Mais, arrivons-en à la région des Alpes, Dauphine et Savoie, qui nous intéresse tout particulièrement dans cette étude.

Le plus ancien document que nous ayons, prouve que dès le vule siècle en Dauphiné, la forge ou martinet est mue par la force des eaux. C'est le testament de Patrice Amion. daté de 736, disant que «son forgeron Majorien a ses artifices mus par les eaux de la Bourbe ». II ast notoire que pendant toute la période $\mathrm{d}$ régime féodal, le moulin appartenait aux seigneurs ou aux moines qui, pour leur usağe, prélevaient redevances. Et, je me suis souvent demandé en examinant la carte d'expansion des moines noirs de Cluny, dans le sud-est, si ce ne serait pas à des fins d'utilisation des forces hvdrauliques que l'on rencontre leur présence, en ces points singuliers de nos torrents alpins : à Domène, dès le $\mathrm{xI}^{\prime}$ siècle, à Vizille ef à SaintPierle-d'Allevard des le xn", et aussi, vers la même époque, au Bourget-du-Lac, jouxte aux martinets de Bourdeau!

Cette remarque se trouve également vraie pour les Chartreux que l'on trouve installés, dès le xnc siecle, aux Courreries de Saint-Laurent-du- 
le Bréda, à Saint-Hugon sur le Bens, et à Aillon. en Bauges sur le Chéran, dès 1184.

Je ne saurais manquer de signalér à ce sajet que dans la correspondance et les écrits de Pierne-te-Vénérable (1122-1156), célèbre abbí de Cluny - qui sont, comme nous le savons, les meilleures p'roductions que nous ait laissé le xrye siecle - il est un document d'une valeur. insigne, celui où le grand abbé, résumant une visite qu'il fit en 1138, aux premiers pères du désert de Chartreuse, dit :

« Les Chartreux, dès leur arrivée au désert. * ont joint aux produits de la terre la pratique " mystérieuse de l'élaboration du fer en faisant "valoir des artifices de deux usines à feu dépen« dantes des courreries. "

Et c'est encore de Cluny que nous vient l'unique texte contemporain :

«Il y a dans le diocèse de Grenoble - et par « conséquent dans celui de Chambéry - un lien " riche en filons de mine de fer, que les habj«tants des montagnes retirent à grande sueur.»

De là, on a appelé Ferrière le lieu qu'habitent ces hommes. Le martinet, colossal marteau actionné par les eaux du torrent, a donné son nom à la fabrique de fer.

Les épéeries de la Fure sont renommées depuis Je x ${ }^{e}$ siècle, celles de Bourdeau, en Savoie, citées par MonTinns « les épées de grand bruit »le sont également et les produits métallurgiques de la province de Viennois des Chartreux sont recherchés par la chevalerie francaise. Aussi, lorsquen 1123, saint Bernard mobilise les armées de la deuxième croisade, il se rend à la Crande-Chartreuse. Ce n'est certes pas un appui moral, qu'il vient chercher auprès de ses frères de nos montagnes mais, comme l'a dit Auguste Bouchayer : "le fer renommé des Alpes du Viennois qui coule des fourneaux cartusiens, l'acier. les armes qui se facomnent sous les lourds et bruyants martinets mus par la force des torrents alpins. »

Il faut bien remarquer que ces forges anciennes, étaient à la fois des usines d'affinage et de forgeage. Les aciéries modernes ont tué l'affinage antique, mais toutes les forges n'ont pas disparu.

Certaines ont gardé, comme les martinets du Bréda, du Bens, du Joudron et de la Fure, des outillages et des traditions où l'on trouve encore la marque des siècles passés. Mais nous verrons par la suite que le xix siècle apportera, avec l'intervention de la houille blanche, une nouvelie phase de l'évolution métallurgique dans ies Alpes francaives, c'est l"aménagement métho. dique des fortes ressources hydrauliques de nos montagnes et leur utilisation sous forme d'énergie electrique, permettant le traitement des minerais les p'us difficiles à réduire.

Ce sera le rôle de l'électrométallurgie que la Savoie verra maîlre dans la Maurienne, à La Pra et sur l'Arly, à Venthon.

\section{LA NAISSANCE DE LA HOUILLE BLANCHE}

Jusqu’a la fin du xixe siècle, les roues de mou in sont courantes dans la région des Alpes, et lon évalue à environ $2.000 \mathrm{cv}$ la puissance totale des roues et rouets, qui tournaient en Dauphiné et en Savoie, en 1832, date de l'invention de la turbine par Fourneyrox.

Cette puissance sera de l'ordre de $4.500 \mathrm{cv}$, en Savoie au moment de l'annexion (1860), de 7.400 ev, en 1882 , sur un total d'envirois $58.000 \mathrm{cv}$, pour la région des Alpes.

C'est le français Benoît Fourneyron, ingénieur stéphanois, inventeur de la turbine hydraulique ct de la conduite forcée qui a aménagé en 1837 la première chute d'eau en Europe, celle de 112 mètres, à Saint-Blaise dans le grand duché de Bade.

Puis, en 1848, est fondée à Grenoble la Maison Ioxa, constructeur-chaudronnier qui construit les premières conduites forcées des Alpes. Joy A, équipe en effel, en 1863, chez MM. Vicat ex Cus à Uriage une chute de 80 mètres, cette chute fonctionnait encore ces dernières années; elle est considérée comme la première en date en Dauphiné et probablement aussi en France.

Et, c'est dans la vallée du Grésivaudan, au débouché de ces vallées suspendues du massif de Belledonne, qu'apparaissent à partir de 1869 , les premiers équipements importants.

Ce sont ceux de :

Bergles à Lancey (1869);

Frever à Brignoud (1871);

Matessibus à Domène (1878).

Ces pionniers étaient des papetiers qui installèrent des chutes en vue de l'utilisation sur place de la force motrice à la fabrication de la pâte de bois.

L'origine de l'utilisation des forces hydrauliques en France est donc papetière et nous verrons qu'il en sera de même en Savoie.

Le vocable de «Houille Blanche » a failli revenir au grand ministre piémontais Cavouk qui, a-t-on dit, aurait le premier prononcé le mol, au cours d'un banquet à Turin, quelques années avant l'amnexion! CAvoun, aurait à cette époque fait allusion aux sources d'énergie contenues dans nos glaciers savoyards, sources perdues 


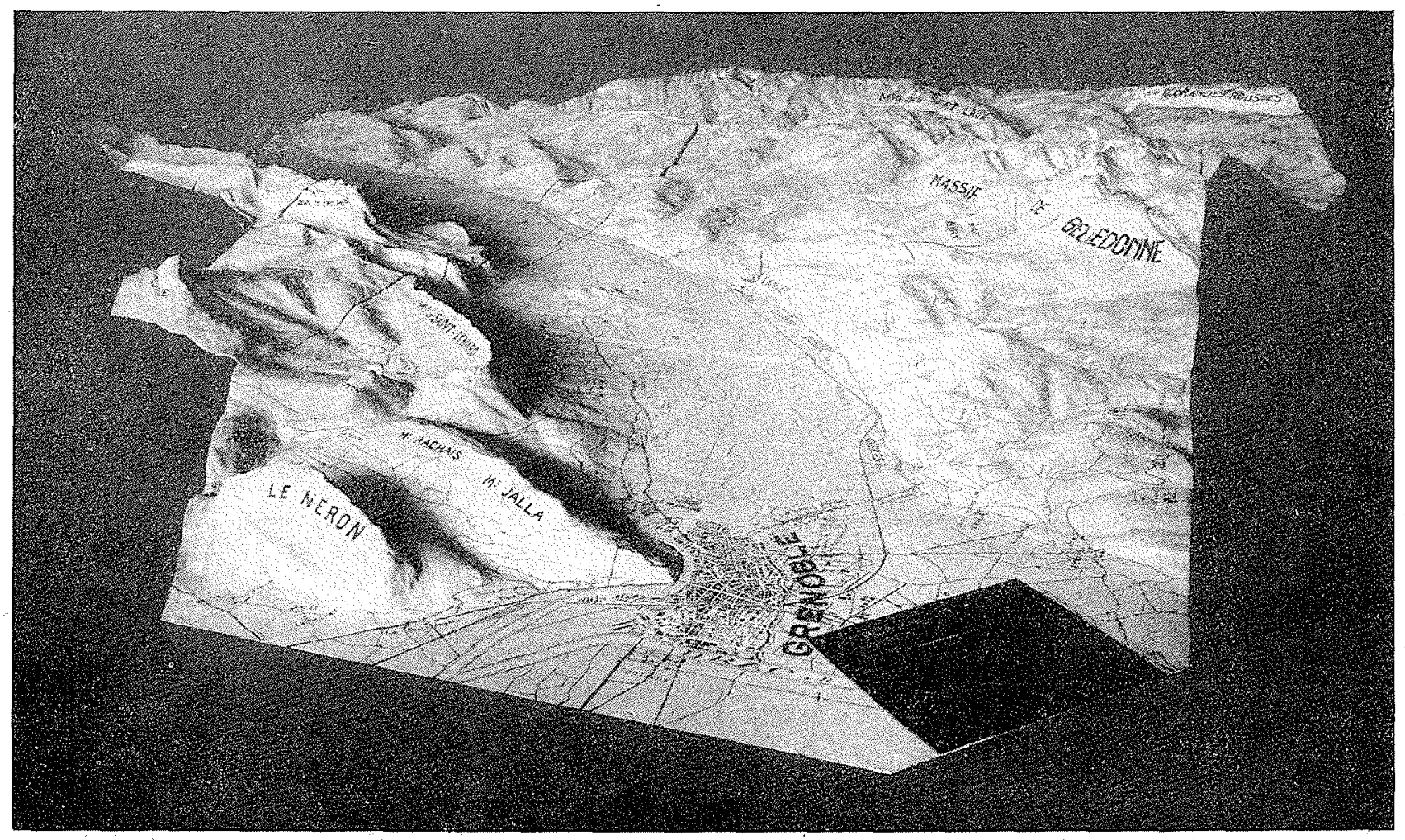

MAUUETE D'ARISTME BERGìs

pour le Piémont par l'annexion de la Savoie à la France. Or, si CAvour a bien pu pressentir la richesse des forces naturelles contenues dans nos chutes savoyardes, quelques deux lustres avant leur première captation en Dauphiné (1869), il est bien prouvé, depuis nos recherches sur les origines de la houille blanche(1) et surtout par celles faites en 1925 par le proferseur Mrrande (z), que c'est à Aristide Bargès qu'est dû le vocable el qui mieux est, lui fit faine fortune.

Aristide Brorils est incontestablement le pere de la houile blanche (3). Ingénieur complet, i!

(1) Voir La Honille Blanche (V. Sraveirne) Elitions Arthaud-Rey, Grenoble, 1925.

(2) Communication de M. MrRaxue, à la Socicte Scienlifigue da Dauphine, 1925.

(3) L'xuvre de Bergès n'a jamais été révelés dans toute son ampleur. Sa gloire est d'aroir préru tous les bienfaits que les grandes forces hydrauliques allaient apporter a l'humanite ; de s'ête attache le premier a l'étude do régime des cours d'eau et des laes de montagne et enfin d'avoir consacré la plus belle partic de sa vie a proclamer les mérites de la richesse hydraulique et a vulgariser les moyens de la conquérir. (E. Dusalaer, comm. Société Scientifigue Dauphine, juin 1945 ; Les pionniers de la Tonille Blanche). installa, non seulement à Lancey (1869) la première chute de France $(200 \mathrm{~m}$.), mais il concut lui-même ses turbines et ses défibreurs pour l'utilisation directe de sa chute. Il perça le premier des lacs de haute altitude (lac de Belledonne à 25 metres de profondeur) en vue de la régularisation du débit de ses chutes; il se fit distributeur d'éclairage électrique dans la vallée du Grésivaudan, inaugurant en 1898, un réseau monophasé à 10.000 volts.

Il dressa le premier inventaire des forces hydrauliques francaises en comparaison avec son bassin de Lancey. Il évalua à environ 10 millions de chevaux (c'etaient alors des chevaux sauvages) la puissance de nos ressources hydrauliques en invitant instamment les pouvoirs publics à en organisez sans tarder l'utilisation! Songeons que Benges lancait cet appel dès l'année 1870 ! et qu'en fait d'évaluation de nos ressources hydrauliques, on n'a guère fait mieux depuis, malgré la précision des mesures des débits et des hauteurs de chute de nos cours d'eau.

Voici ce que dit Bergis dans la mémorable note publiée en 1889, à l'occasion de l'Exposition Universelle de Paris : 
«Il semble que le moindre filet d'eau, dan. « les grandes hauteurs, n'est plus de l'eau, mais « de la houille noire qui sourd automatiquement "du sol, et alors le nom de «houille blanche" "pour baptiser ces richesses vient naturellement « à l'esprit.

« De la houille blanche, dans tout cela, il n'y « en a pas; ce n'est évidemment qu'une méta« phore, mais je n'ai voulu employer ce mot que " pour frapper l'imagination et signaler avec *vivacité que les glaciers et les montagnes « peuvent être exploités en forces motrices, être * pour la région et pour l'Etat des richesses "aussi précieuses que la houille des profon« deurs. »

On ne saurait mieux dire et mieux prédire pour un pays comme le nòlre, si pauve en houile noire et cest à juste titre qu'Aristido Brngiss a été consacré le père de la houille blanche.

Les premieres chutes, utilisant sur place l'énergie hydraulique, étaient à peine installées, que successivement les français :

Grsmms, ouvier de génie, invente la dynamo dectrique à courant continu (1870) ;

Foxtane, à l'Exposition de Vienne (1873) révele le principe de la réversibilité d'ane dynamo, c'est-à-dire son fonctionnement en moteur lorsqu'elle est alimentée par un courant électrique ;

Deprez Marcel, sous I'initiative d'Edouard Rey, maire de Grenoble, réalise, en aout 1883, les célèbres expériences de transport de force par l'électricité, entre Jarrie-Vizille et la halle de Grenoble.

La date de 1883 est une date mémorable dans I'histoire industrielle des Alpes, car elle marque le point de départ d'une véritable transformation. Ce jour-là, le principe de la marche en moteur de machines dynamo-électriques recevait une éclatante démonstration, et il devenait possible aux industriels de ne plus etre astreints a consommer su'r place l'énergie produite par les chutes, comme cela se faisait, depuis toujours, avec les vieilles roues datant du début de l"ère chrétienne et par les turbines qui venaient de naitre. Rappelons qu'Aristide BErgiss faisait partie du Conseil Municipal de Grenoble l'année de ces mémorables expériences et qu'il les suivit avec une passion d'inventeur et de novateur. Il le prouvera par les propositions qu'il fit, en 1899, a la ville de Grenoble pourr lui fournir la lumière, la force et la chaleur par l'électricité produite par ses chutes du massif de Belledonne (1).

A partir de cette date, c'est la grande envolée car, gràce à l'invention du kansformateur satique, par l'infortuné francais GauLARs, et des machines génératrices à courants alternatifs, le transport de l'énergie des chutes allait devenir plus facile qu'avec le courant continu des dynamos Gramme et Marcel Deprez.

Et je suis particulièrement heureux de rappeler ici, dans cette revue « La Houille Blanche » rénovée, que cest notre compatriote savoyard, l'ingénieur E. Dusavgsy, l'apòtre de l'emploi de J'aluminium dans les càbles de distribution, qui a réalisé en 1898, le premier transport d'énergie à courants $t r i p h a s \dot{s}$ 15.000 volts, entre Engins et Voiron.

Nous savons tous que les transports d'énergie dectrique se font aujourd'hui à de très hautes tensions $120-150$ et 220.000 volts, à courants alternatifs, tensions permetlant de transporter à des distances de 400 à 500 kilometres des puissances de l'ordre de 100.000 chevaux. Les nouvelles lignes de transport Alpes-Paris, sont prévues à la tension de 400.000 volts.

Voila le chemin parcouru dans les Alpes, depuis 1898 !

\section{LA SAVOIE ET LA HOUILLE BLANCHE}

Comment et par qui s'y est fait l'aménagement des chutes?

«Il y a bien peu d'années encore, les torrents « de nos montagnes faisaient le bonheur des « artistes, mais nul ne pouvait se douter que « la splendide ruée des eaux bouillonnantes « deviendrait pour notre pays une inépuisable « richosse. »

\section{(A. BonRel, 1925).}

Voilà ce que disait, en 1925, notre ami, Antoine BonRri, alors Ministre des Forces Hydrauliques, dans la magnifique préface qu'ii a bien voulu consacrer à notre ouvrage sur la houille blanche.

Ainsi donc, tout était prêt en Dauphiné entro 1869 et 1890 , et la Savoie ne devait pas tardel

(1) Bientot, le pays de la houille blanche, puis la Franes entiere se courrirent de ces jnnombrables réseaus qui apportent l'énergie mécanífue aux plus puissantes usines, comme la lumière aux plus humbles demeures. La mourement est done bien parti du Dauphiné, l'élan a bien éte domné par les pionniers dauphinois. Note do M. Dusarger, sur Bargès et Jes Pionniers de la Honille Blanche, 1945 ) 
à mettre à son tour en valeur les remarquables dispositions naturelles de ses torrents : abondance des eaux, grosses précipitations (Châblais Beauges - Mont-Blanc), régularite des régimes, fortes pentes et forts débits en Maurienne et Tarentaise, présence de vallées suspendues dues a l'action glaciaire (Bissorte et La Girotte), verrous et ombilies (Tignes), enfin, lacs de haute altitude.

A l'exemple du Grésivaudan elle utilisera la technique alpine (r) de ses vallées suspendues, en utilisant sur place l'énergie dans une usine de transformation.

L'emplacement sera naturellement choisi dans les vallées parcou'rues par la voie ferrée et, fait typique, c'est le savoyard Joseph Hortevr, maître de postes aux Chavannes (Maurienne) au lieu dit «La Grande Maison » sur la route ParisRome, tenant là, un grand relais de chevaux et auberge qui, dès l'apparition du rail en Maurienne, liquide ses chevaux domestiques pour equiper à Saint-Rémy (en 1876) une chute de $300 \mathrm{cv}$, hateur 60 mètres, en vue de défibrage du bois.

Horteur équipe celte chute en association avec un ingénieur M. Moré qui était en relation avec Aristide Benges, de Lancey, pour ses défibreurs.

Donc, comme en Dauphiné et suivant d'un lustre les pionniers du Grésivaudan, c'est un papetiér qui ouvre le feu en Savoie, en équipant la première chute à Saint-Rémy (1876).

Puis, encore en Maurienne, en 1885, c'est ia chute du Chamaix, a Fourneaux.

Et voici 1889 , la première chute importante par son débit $2 \mathrm{~m}^{3} / \mathrm{s}$, hauteuir $60 \mathrm{~m}$., équipée à Venthon sur le Doron-de-Beaufort par un papetier, M. Aunru. Celui-ci installe à Venthon une papeterie avec fabrication de la cellulose, fabrication semblable à celle qu'il avait déjá montée en 1884 à la papeterie de $\mathrm{M}$. Vieluomme à Domène.

Et, montrant comment l'histoire industrielle s'enchaine, c'est VielHomme qui installera, en 1888, à Froges, une chute pour la fabrication de l'aluminium en collaboration avec les ingénieurs Heroult et Toussaine et créera la Société Electrométallurgique de Froges qui, en 1892, ira s'installér en Maurienne, à La Praz.

C'est a Venthon que Paul Grop commencera en 1898, dans un local loué à Aubry, ses recherches électro-métallurgiques qui le conduiront en 1903, à créer à Ugine la Société Electrométallurgique Paul Grrod, qui deviendra,

(1) Désignation due à Raoul Brancharo, ainsi que celles de Pyrénéenne, Massif Central et en Savoic, technique de l'Are et de la Girotte. comme nous le verrons bientôt, l'aciérie électrique la plus puissante d'Europe, absorbant dès 1938, une puissance de l"ordre de $100.000 \mathrm{cv}$. C'esi aujourd'hui la Société d'Electrochimie et des Aciéries Electriques d'Ugine, et Venthon reste le berceau de l'électro-métallurgie française.

Notons ici, à l'actif éclairage électrique en Savoie que la Roche-sur-Foron (1885) et Bridesles-Bains (1890) sont les premières à posséder la lumière électrique. Chambéry ne l'aura qu'en 1894, par l'équipement de la chute de $650 \mathrm{~m}$. du Cernon $(600 \mathrm{kw})$, renforcée en 1909 par le Bréda $(1.500 \mathrm{kw})$ et par le Bens en 1919 $(6.500 \mathrm{kw})$.

La phase papetière ayant montré la voie, voici que s'ouvre à partir de 1890 la phase de l'électrométallurgie.

Ciest toujours en Maurienne qu'elle apparaît:

1890 Saint-Colomban, fabrique de carbure;

1890 Calypso, sur la Valloirette.

Calypso est fondée en 1890 par les frères Bernari) de Paris, l'ingénieur Mrnet étant directeur. Cette usine passera par la suite à la Société Parisienne d'Aluminium, puis en 1896, à l'important groupe Péchiney.

Calypso est la première en date qui apparait en Maurienne et dans le monde pour la fabrication de l'aluminium. Elle a donné son nom aux lingots d'aluminium fabriqués par la Cie Alais, Froges er Camargue. J'avais longtemps pensé que le nom de Calypso était dû̀ I'imagination de Tristan Bernaro, neveu des fondateurs, qui aurait pu entrevoir dans cel antre électrométallurgique serti à la tombée des sombres gorges de la Valloirette, un lieu Iuj rappelant la grotte de Calypso. Eh bien! non, l'origine du nom est plus originale et plus populaire. Son auteur serait un mauriennais, un Michot, boulanger de Saint-Michel-de-Maurienne, heureux propriétaire d'une vigne situce sur l'emplacement actuel de l'usine, qui disait toujours « je vais à ma vigne à Calypso 》 alors qu'en réalité le lieu dit s'appelait du nom, moins noble «les gorets » du fait de l'existence d'une porcherie à proximité (x).

C'est à cette époque (1891) que survient, avec l'équipement de l'Arc à gros débit $(52 \mathrm{~m} 3 / \mathrm{s}$ à Hermillion) et à grande déclivité $(570 \mathrm{~m}$. entre la Chambre et Fourneaux) une technique nouvelle, celle de la prise en rivière par barrage avec dérivation en tunnel et descente su'r l'usine par conduite forcée en tôle d'acier à gros diamètre. Cette technique a été retenue par Raoul Branchard sous le nom suggestif de technique de l'Arc.

(1) Communiqué par. M. Jouannet directeur de l'usino de la Saussaz à Saint- 
Ce sont là, sur l'Arc, des chutes de moyenne hauteur, de 40 à 80 mètres, mais à forts débits.

1891 Prémont, chute $75 \mathrm{~m}$.

1892 La Praz, chute $35 \mathrm{~m}$. portée à $83 \mathrm{~m}$., en 1898.

1903 La Saussaz, chute $74 \mathrm{~m}$.

1908 Les Plans, Saint-Jean-de-Maurienne, chute $67 \mathrm{~m}$.

1912 Pontamafrey, chute $38 \mathrm{~m}$.

1913 Montricher, chute $41 \mathrm{~m}$.

C'est à l'usine de La Plaz que l'ingénieur Hénouls, promoteur de la fabrication électro. lytique de laluminium, proposa et réalisa en \$s98 la solution originale d'enjamber la rivière avec une conduite forcée, en forme d'are surbaissé, dite passerelle syphon.

C'est également à La P'raz qu'HéroulT monta, pour le compte de Froges, la première fabrication industrielle de l'aluminium et qu'il fit, en mars 1900 , ses premiers essais d'acier au four électrique.

En 1902, lors de la célèbre visite à La Praz

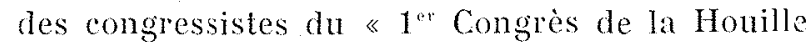
Blanche », Héroutu effectua devant eux la premicre coulée publique d'acier au four électrique, une coulée de deux tonnes.

Il est à retenir que la première industrie électrométallurgique qui utilisa la houille blancho est celle de l'aluminium et qu'elle fut réalisce, en Savoie, à La Praz, en 1892.

Comme l'a dit M. Flusin, directeur de l'Instilut d'Electrochimie de Grenoble :

« Le nom d'Henout sert désormais à qua" lifier, sans qu'il soit besoin de préciser davan" lage, deux des plus grandes conquêtes de « l'électrolyse et de l'électrothermie : le procédś "Hrizorta universellement employé p o u $\mathrm{r}$ «l'extraction de l'aluminium par électrolyso « et le four Hénourt, l'appareil électrother« mique le plus répandu pour la production de "l'acier au four électrique. »

Aujourd'hui, la Maurienne, avee ses usines de La Praz, La Saussaz, Prémont et des Plans, produit annuellement environ 35.000 tonnes

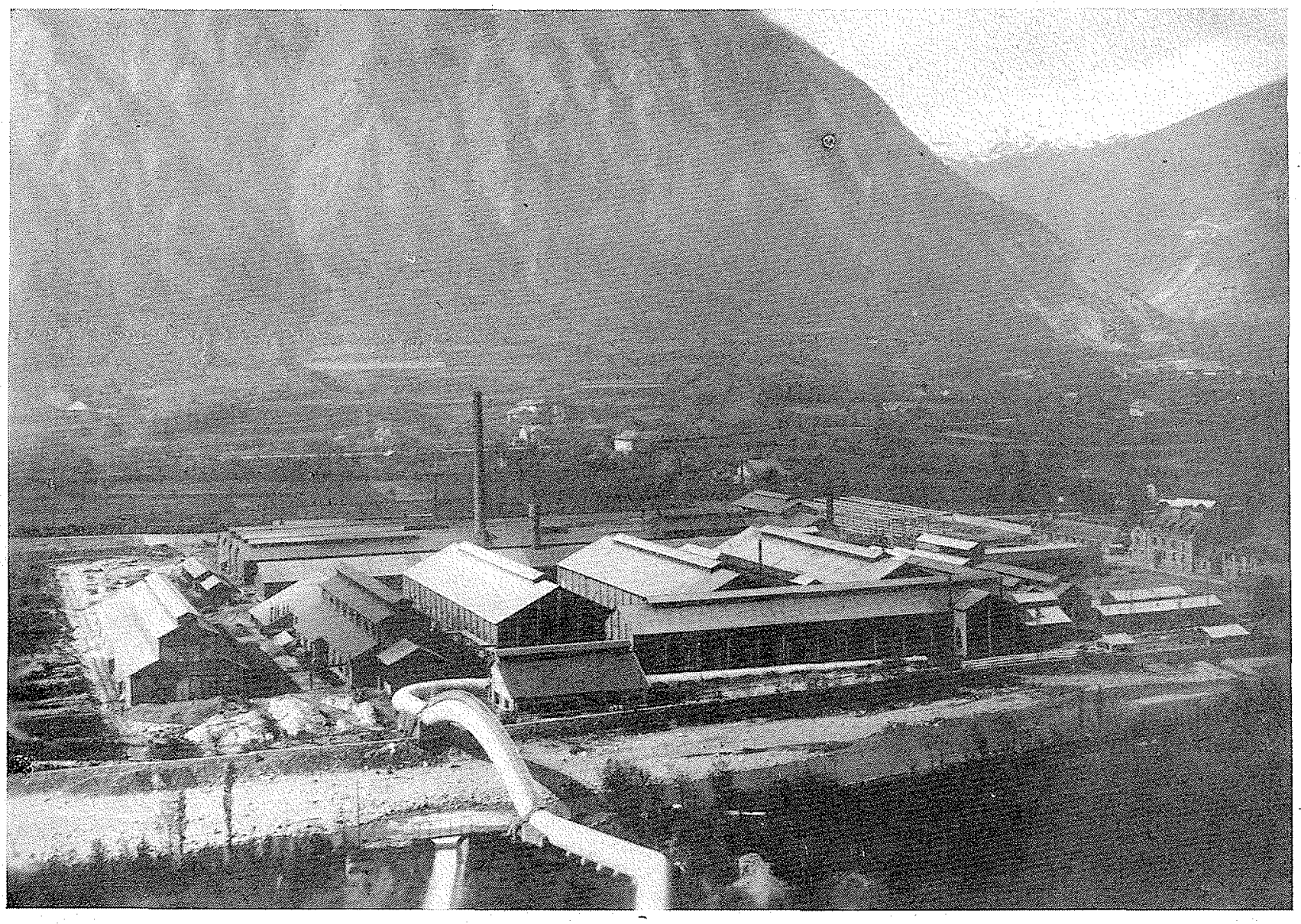

COMPAGNIE ALAIS, FROGES ET CAMARGUE. - USINE DES PLANS A SAINT-JEAN-DE-MAURIENNE (Traversée de lite par conduitr-syphon) 


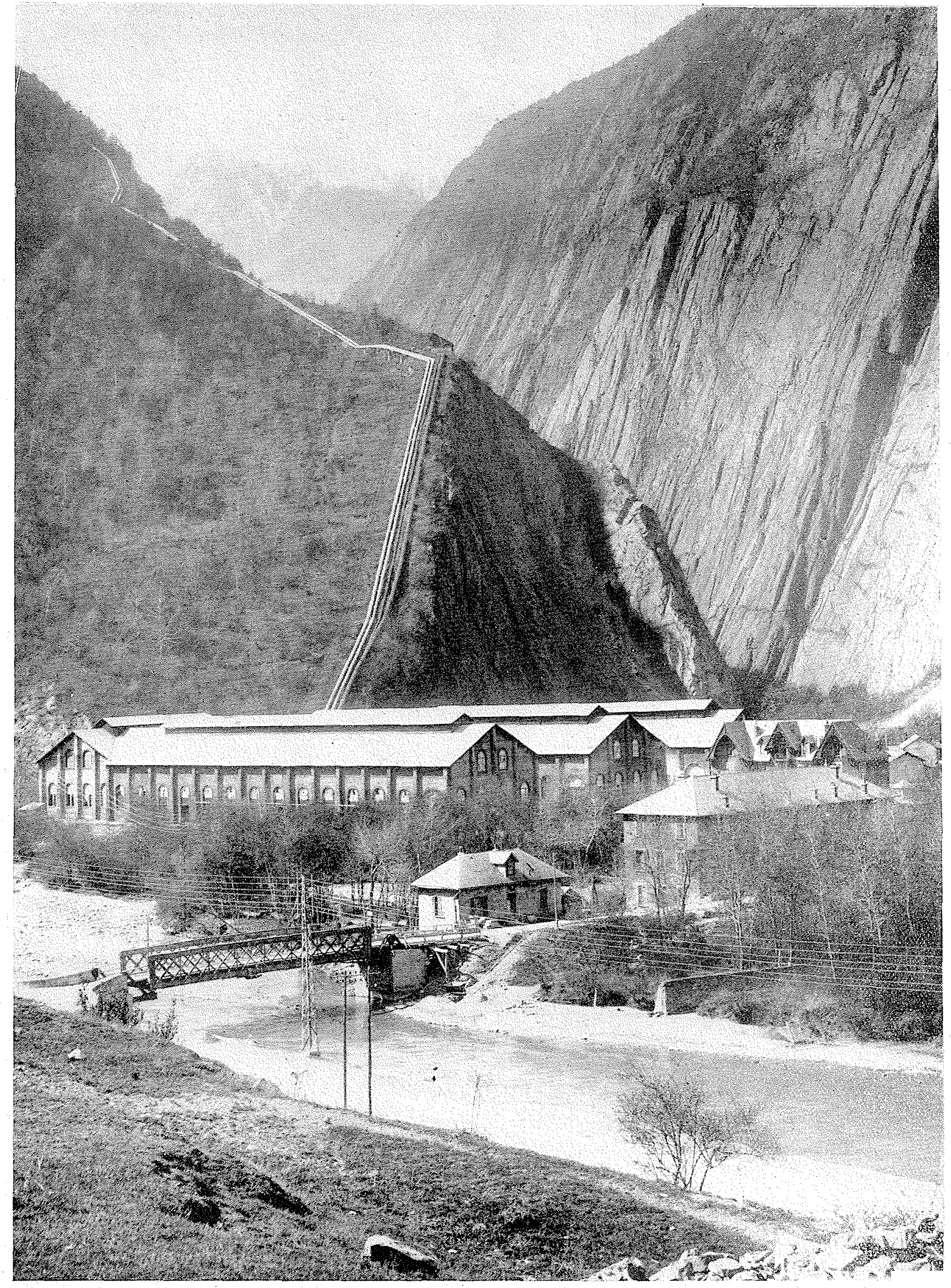

CALYPSO, - IA PRIMIÉRE GRANDE USINE D'ALUMINI UM DE la MAURIENNE (1891) 
d'aluminium, dont 21.000 tomnes pour la seule usine des Plans à Saint-Jean, tonnage représentant $40 \%$ de la production francaise en ce métal.

La Maurienne, « ce val de l'aluminium », ce berceau de l'électrolyse et de l'électrothermie, aturait dù être baptisée :

\section{LE VAL D'HÉROULT}

et la statue de ce grand ingénieur français, statue en aluminium doré devrait s'élever sur l'Arc, à l'entrée de la Maurienne ! (1)

Notons ici, en Matrienne, que la fabrication du carbure y est le brillant second de celle de l'aluminium car, les usines de Glandon, Argentine, Saint-Gobain, Montricher el la Pouille, ont produit environ 35.000 tonnes de carbure en 1941.

Gràce à ces puissants équipements hydroélectriques sur l'Arc, en Maurienne, la Savoie prend des lors une certaine avance sur le Dauphiné, car les grandes usines de la Société Electrochimique de la Romanche à Livet (chute $60 \mathrm{~m}$., débit $8 \mathrm{~m}^{3} / \mathrm{sec}$.) sont postérieures à 1898 et n'arriveront à un slade vrament industrici que vers 1902, sous l'énergique action de CharlesAlbert Kelter.

Comme l'a fort intelligemment fait remarquer Raoul Blanchard, au cours de sa belle conférence de Chambéry, en juin 1943 , cette avance, la Savoie la gardera, gràce à l'apport des équipements de ses autres vallées, dès que le rail y apparaitra.

1893 Saint-Béron sur le Guiers;

1895 Les Chavants-Servoz, sur la ligne de Chamonix ;

1896 Chedde sur l'Alve supérieur.

Chedde, créé par Georges Bergiss, (fils d'Arislide BEngìs, père de la houille blanche) et par MM. Coprin et Hippolyte Bouchayer, des noms bien connus, cette chute a conservé longtemps le record du cheval installé, au meilleur marché, à 100 francs le ev contre 5 à 6.000 de nos jours!

(1) La France, si prodigue de ses grands hommes, si oublicuse de la gloire de ses enfants, devait dams son axtrême misere reconnaitre ses gloires passés. Des compagnies, comme la notre, fouillant l'histoire de leur province devaient lui rappeler qu'il est des gloire: ou'on n'oublie pas ! Aussi, Messieurs, je demande a notro président, qu'à défaut d'une statue érigée à l'entrée do la Maurienne - erection qui sera peut-être assure pai les soins d'Alais, Froges et Camargue, dont Herour's fait In fortune - Ia Societe Savoisienne d'Histoire, suggere à la capitale du duché de donner le nom d'Féroult à la principale place du nouveau Chambéry d que la biographie a cet ingénieur illustre prenne place dans tous les manuels scolaires de la Savoie. (Histoire de la Honille blanche en Savoie, Communications des 17 juin et 7 octobre 1945 , de V. Sravestre à la Sociéle Savoisienne a'Histoire).
1899 Le Giflre en Faucigny, créé par te savoyard BARUT;

Puis, c'est le tour de la Tarentaise, où la voic lerrce apparaît en 1893 ;

1896 Notre-Dame - de - Briançon, sur l'EauRousse,

Chute de $230 \mathrm{~m}$. équipée par Henri Gall, fondateur de la Société d'Electrochimie, usine qui disposera par la suite, du fait des apporl; succesifs de centrales hydroélectriques equipées sur les Doron de Belleville et de Bozel, d'une puissance de l'ordre de $20.000 \mathrm{kw}$.

On utilise là, en Tarentaise, les chutes des vallées suspendues affluentes paree que la vallée principale n'a pas assez de pente.

Ce sont :

1898 La Rageat (1) sur le Doron de Belleville ;

1899 Le Villard, chute 240 m., équipée par la Compagnie Fives-Lille ;

1906 La Pomblicre, près Moùtiers ;

1911 La Rosière, de Saint-Bon ;

1912 Doron de Champagny, 540 m., chutes jumelles ;

1928 Vignotan, $192 \mathrm{~m}$., sur le Doron de Bozel.

La chute de Vignotan, étant équipée pour $21.000 \mathrm{kw}$, ]a puissance du Doron de Bozel atteint $43.000 \mathrm{kw}$, portée à 50.000 avec Balendas, le groupe Tarin atteindra $130.000 \mathrm{kw}$ avee les travaux en cours.

A partir de 1900 , le développement de la production et du lransport de l'énergie par fil va permettre l'utilisation des chutes situées à l'écar̆ des vallées principales et de se libérer de la tyrannie du rail. On assiste alors en Savoie à un veritable épanouissement de centrales hydroélectriques :

Bomnevaux et Chevenoz, du groupe des Dranses ;

Brassilly, Chavaroche et Vallières du groupe du Fiers;

La Bridoire, sur le Tiers (1910) écoulement da lac d'Aiguebellette, avec utilisation d'une tranche de 6.000 .000 de $\mathrm{m}^{3}$ de ee lac.

Puis apparaissent :

1911 La Rosière, sur le Doron de Bozel;

(1) Cest a cette centrale de La Rageat qu'a débule notre compatriote ef ami Honore Gluso, dont on fetait le 3 février 1945, dans le grand amphithéatre de l'Institut. la belle carriere d'Ingénieur en chel des Services «Exploitation » de la Sociéte Genérale de Foree et Lumière. 


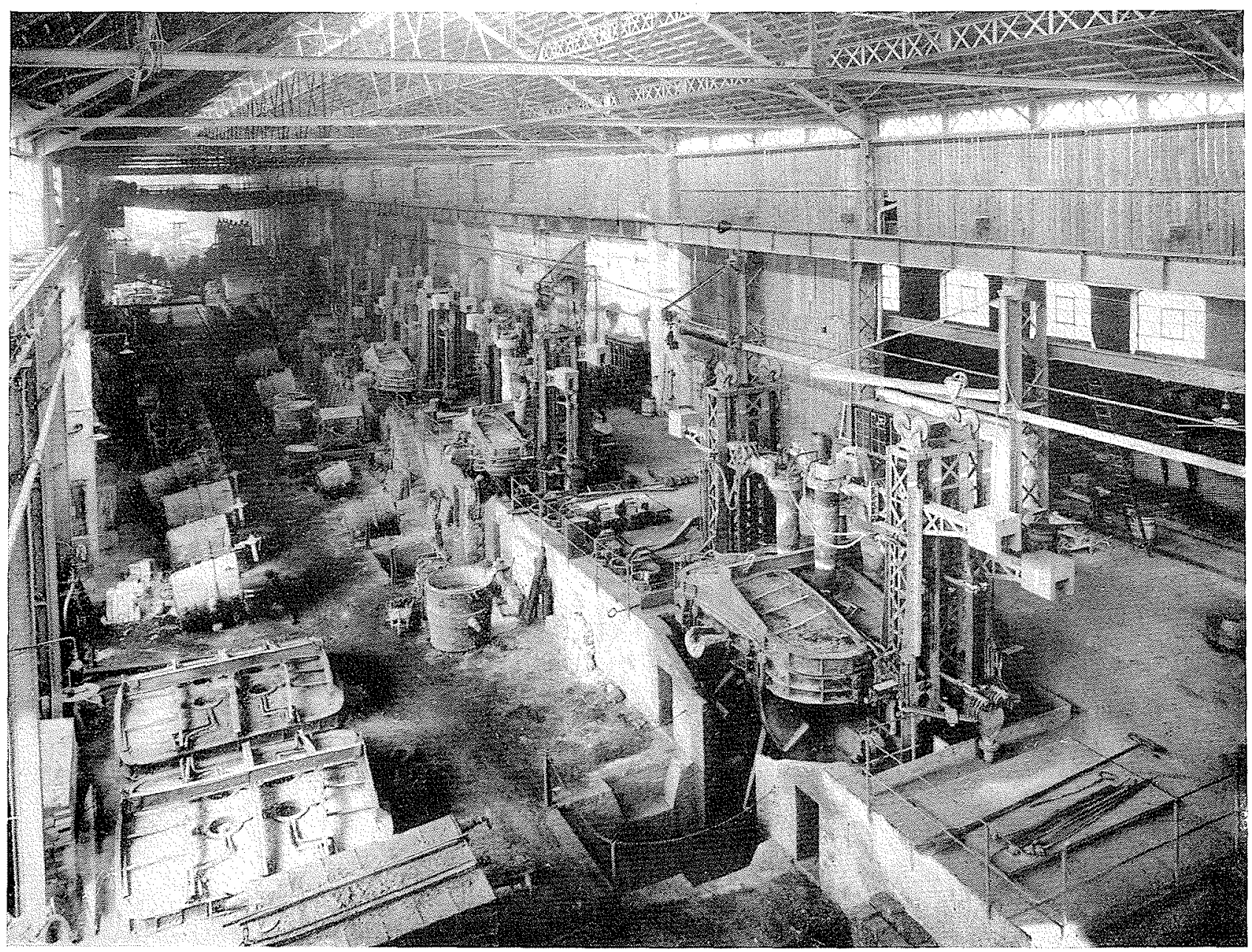

SOCIÉTÉ D'ÉLECTROCHIMIE ET ACUÉRIES ÉLECTRIQUES D'UGINE Batierie des fours électriques

1919 Le Bens, à Arvillard (alimentation Chambéry) ;

1920 Viclaire, sur la haule Iscre, $400 \mathrm{~m}$. $40.000 \mathrm{cr}$.

La Viclaire, avec ses $40.000 \mathrm{cv}$ transportés su: Lyon, a été la plus puissante chute de Savoie juqu'en 1937, année de l’équipement de Bissorte en Maurienne.

Et dans cette vallée de Maurienne, e'est en :

1908 Le Glandon;

1918 Avrieux, de la Compagnie de Sain!Gobain ;

1919 La Neuvache.

Puis, c'est surtout le groupe des Aciéries Electriques d'Ugine, d'un si grand intérêt. Après Venthon, équipé par Aurry en 1889 , la chute des Fontaines, à Ugine, est équipée par Grnod en 1904, suivie par le groupe du Bon Nant :

1908 Fayet ;

1909 Bionnay;

1909 Les Rateaux, groupe complété par le puissant groupe du Doron de Beaufort;

1905 Venthon renforcé ;

1908 Queige ;

1919 Roengers

1920 Belleville, chute de la Girotte, $500 \mathrm{~m}$.

Le Lac de la Girotte régulateur du domaine hydraulique de la Société d'Électrochimie ef Aciéries Électriques d'Ugine (1)

Ia Société d'Electrochimie d'Ugine, poursuivant l'idée de M. Aubry (1904) perce (1923-25) le lac de la Girotte a 80 mètres de profondeur,

(1) La Honille Flanche, mai-juin 1922 : Les usines hydroélectriques de la Cie Paul Girod, à Ugine, par V. Sruvestre.

Troisieme Congrès de la Houille Blanche, Grenoble 1926, tome II, Usines Societé d'Ugine, vallée de Beaufort, p. 1429, travaux pereement lac de la Girotte, p. 1637. 
alimentant sous 500 mòtres de chute la centrale de Belleville en vue de constituer un réservoir saisonnier rigularisant d'une part, toutes les centrales óchelomées sur le Doron de Beaufort, de Belleville à Venthon, sous une chute totale de $1370 \mathrm{~m}$, en fournissant annuellement de 80 a 90 millions de kwh et d'autre part toutes celles de son domaine hydraulique de l'Arly et du Bon Nant, du Bassin de l'Arve.

Le lac de la Girotte a un ombilic de 58 hectares, situé à $1.720 \mathrm{~m}$. d'altitude, le creux est de $99 \mathrm{~m}$. et sa réserve naturelle de 29 millions de $\mathrm{m}^{3}$.

Lapport naturel dans le lac était de 7 millions de $\mathrm{m}^{3}$ seulement, on a complété cet apport frar Jes aménagements suivants :

1923 Aménagement en station de pompage de la centrale de Belleville par deux groupes de $4.000 \mathrm{cv}$ et un de $10.000 \mathrm{cv}$, permettant de refouler dans le lac de 8 a 12 millions de $\mathrm{m}^{3}$ annuellement.

1925 Dérivation du ruisseau de Colombes et

1932 Dérivation du ruisseau

de Rosseletle ....... 2.000.000 de m.3

1939 Dérivation d u Bon

Nant . . . . . . 2 23.000.000 de ms

1944 Dérivation eaux gla-

ciér Tré-la-Tête .... 40.000.000 de m³

La surélévation du lac de la Girotte au moyen d'un barrage, augmentant sa capacité de $13.500 .000 \mathrm{~m}^{3}$ permettra d'y accumuler annuellement de 70 a 75.000 .000 de $\mathrm{m}^{3}$, dont on arrivera a utiliser pendant les six mois de basses eaux jusqu'à 4.0 à 45.000 .000 de $\mathrm{m}^{3}$, pouvant donner un total de 110 à 120.000 .000 de kilowatts-heure, tandis qu'une trentaine de millions de mètres cubes resteront disponibles pour les pointes d'été et d'automne.

\section{Le curieux travail de dérivation des eaux du glacier de Tré-la-Tête massif du Mont-Blanc, dans le lac de la Girotte}

La Société d'Electrochimie et des Aciéries Electriques d'Ugine a entrepris et mené à bien, en pleine période d'occupation (1942-44), le curieux travail de dérivation des eaux du gla-. cier de Tré-la-Tête dans le lac de la Girotte(r).

La dérivation est faite par un tumnel de $5 \mathrm{kms}$, se développant aux pieds des contreforts du Mont-Tondu et allant se raccorder à l'adduction du haut Bon Nant. La cote de la prise d'ean se trouvant fixće à $1.920 \mathrm{~m}$. et la langue términale du glacier étant à $1.900 \mathrm{~m}$. d'altitude, les

(1) Amémagement d'une prise d'cau sous-glaciaire au glacier de Tré-la-Tete. Communication de M. WaEsen, 23 innvier 1943, a la Societé Seientifique du Dauphine. ingénieurs ont été conduits là, à l'aménagement d'une prise d'eau sous-glaciaire.

Le débit dérivé est de l'ordre de 50.000 .000 de $m^{3}$ par an, avec l'avantage insigne d'avoil: un apport dans le lac de la Girotte, d'autant plus fort que lété est plus chaud et plus ser. Notons que les mois de juillet et août, à eux seuls, représentent $50 \%$ du débit annuel du glacier, dont $95 \%$ s'écoule pendant les six mois de la saison chaude.

Les travaux entrepris, des 1941, sous la direction de M. Wasmrn, ingénieur de la Société d'Ugine, ont permis de faire des constatations les plus intéressantes pour les glaciologues : temperature sous le glacier, contact de la glace avec la roche encaissante, régime du torrent sous-glaciaire, mode d'écoulement de la glace dans la gorge sous-glaciaire, ete...

Le $1^{\text {or }}$ juillet 1944 , les eaux glaciaires de Trila-Tête se déversaient dans le lac de la Girotte et passaient pour la première fois, du bassin de l'Arve à celui de l'Iscre :

C'est bien le travail le plus original qui ait été exécuté dans les Alpes pendant la guérre que celui de la dérivation d'un glacier dans un lac de haute altitude en vue d'une accumulation d'énergie de régularisation des u'sines hydroélectriques de tout un ensemble de bassins.

Tout cet ensemble du domaine hydroélectricue de la Société d'Ugine, représentant quelques $120.000 \mathrm{kw}$ installés fournissant une énergie totale annuelle de 450 millions de kwh, atteignant 510 millions lorsque le lac de la Girotte sera surélevé pour emmagasiner les apports complets de 'Tré-la-Tête, est l'un des plus puissants et des plus complets des Alpes $\left(1 / 6^{\circ}\right.$ environ de la Savoie) et le plus beau fleuron apporté a la couronne savoyarde de la houille blanche.

\section{Bissorte, la plus haute chute de France 1150 mètres - $120.000 \mathrm{cv}$}

Et puis nous arrivons à la plus puissante chute de France, la dernière née en Maurienne, ou comme nous l'avons vu est apparue la première chute équipée en Savoie.

II est remarquable que jusqu'à ces dernières années toute l'énergie du bassin de l'Árc était absorbée sur place aux fabrications électromélallurgiques, aluminium et carbure. Pendant près d'un demi siecle pas un seul kilowatt n'est sorti de la Maurienne!"

Ce n'est que depuis 1937, que la Société Hydroélectrique de Savoie a dissipé ce paradoxe, en équipant une chute puissante destinée à s'interconnecter avec les grandes centrales de l'Isère, de la Romanche et de la Durance et de fournir aussi, sur le réseau général français et 


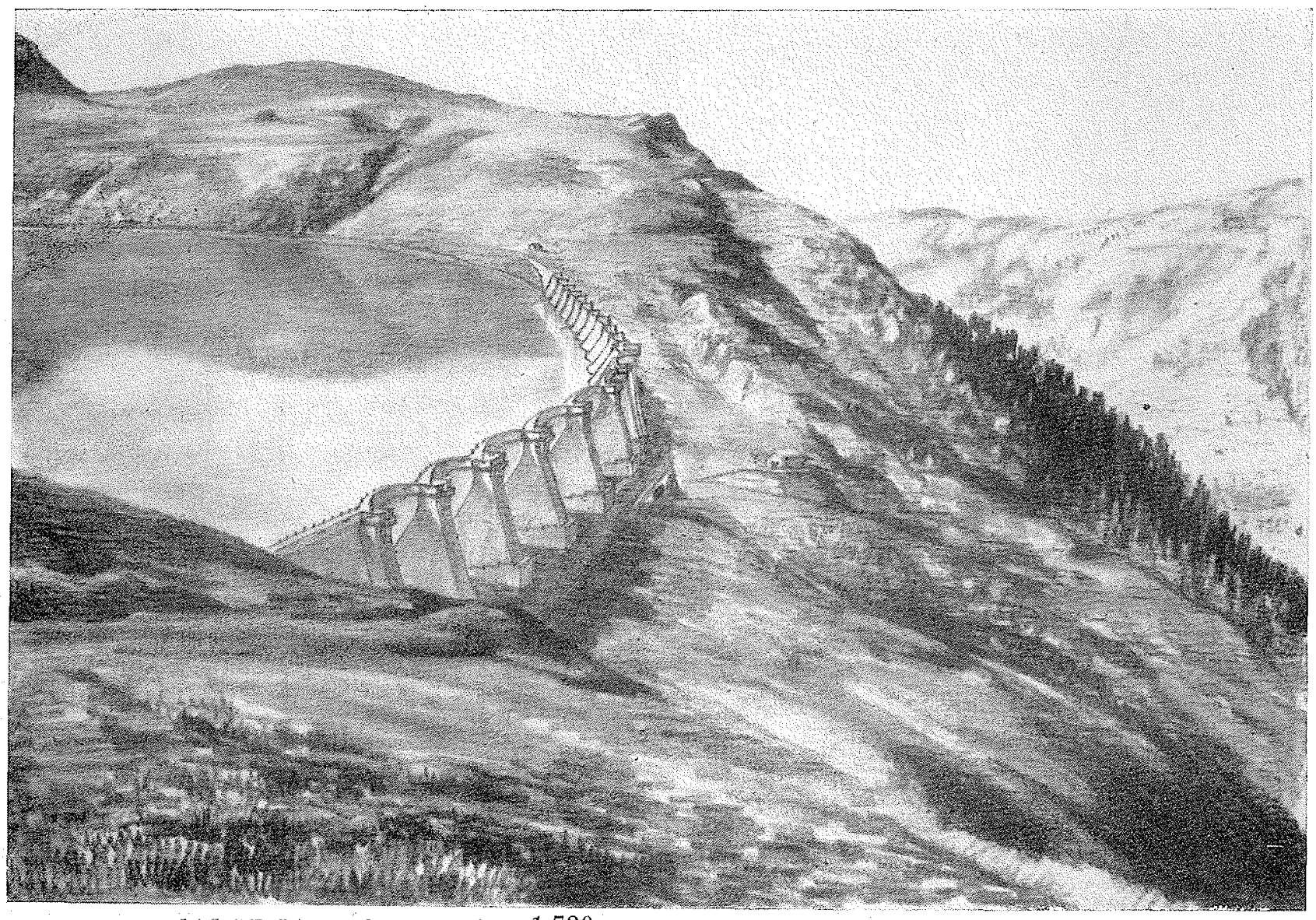

IAC DE LA GIROTTE - COTE $1.720 \mathrm{M}$ - MAQUETTE DU NOUVEAU BARRAGE

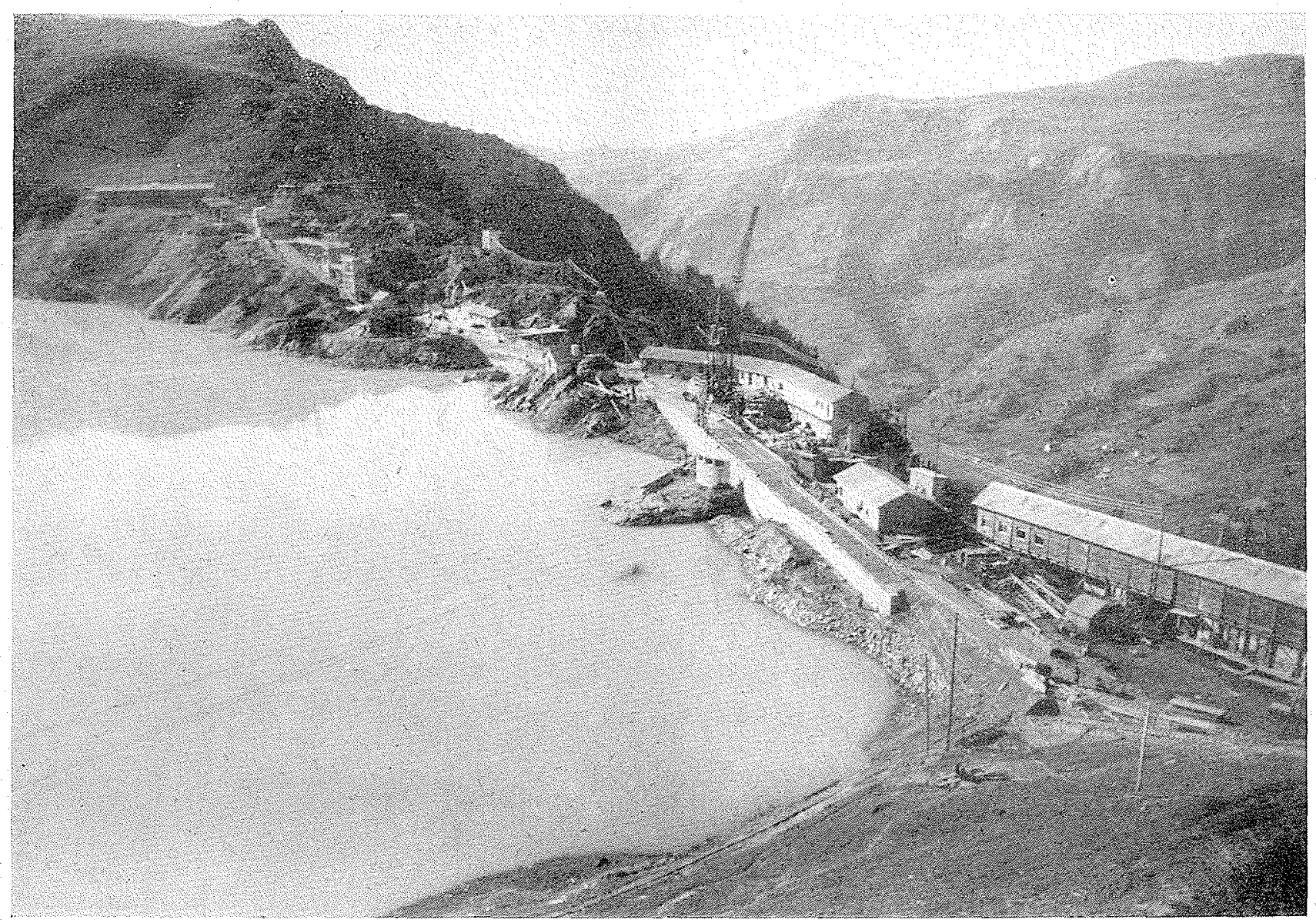

LAC DE LA GIROTTE - COTE 1.720 M. - LES TRAVAUX DE SURÉLÉVATTON DU BARRAGE EN OCTOBHE 1943 
sur Paris, des énergies de pointes particuliere. ment précieuses.

C'est la chute de Bissorte, à La Praz, vis-àvis la chute de 1892 d'Héroult. Il s'agit là, d'après Raoul BLANCHAlld, d'un cas grandiose d'application de la technique pyrénéenne d'utilisation des lacs complétée par celle de la Girotte.

Hauteur de chute 1.150 mètres, puissance installée $120.000 \mathrm{cv}$, la conduite forcée en tube d'aciér auto-fretté, système de l'ingénieur dauphinois G. Fermand, est la plus puissante du monde : $1.400 \mathrm{~m} / \mathrm{m}$ de diamètre, $7,5 \mathrm{~m} 3 / \mathrm{sec}$, puissance 105.000 chevaux contenus dans le même tuyau.

Un barrage de $545 \mathrm{~m}$. de Iongueur, tendu à la cole $2.08+\mathrm{m}$. leconstitue l'ombilic du lac de Bissorte. Celui-ci, d'une capacité de 40 millions de mis, recoit par dérivation en tunnel de $7,5 \mathrm{kms}$ les torrents du Charmaix et de Bonnenait.

Bissorte est actuellement la plus puissante chute de France et la plus haute des Alpes :
1.150 mètres, dépassée seulement en France par celle de $1.413 \mathrm{~m}$, de Portillon, dans les Pyrénées; la plus haute du monde restant celle de Dixence dans le Valais avec ses 1.750 mètres.

Bissorte est a triple effet :

- déployer beaucoup d'énergie lors des pénuries ;

- fournir des pointes de grande puissance, $82.500 \mathrm{kw}$, énérgie précieuse en heures creuses des grands réseaux;

-_ accroître le débit d'étiage de l'Arc - à Calypso, La Saussaz $3.800 \mathrm{kw}$ portés à $5.000 \mathrm{kw}$ -- permettant de garder toute l'annce, même en basses eaux, un grand nombre de fours à aluminium en marche dans la Maurienne et aussi sur la Durance.

La centrale de Bissorte ( 1 ), odieusement saccagée par les nazis lors de leur fuite en Maurienne en aoùt 1944 , est relice aux grandes

(1) Voir La Honille Blanche, n" 1, nov. 1945, la chute de Bissorte, la destruetion de la centrale et sa reconstruction par L. Pousse, avec introduction de M. Colomb, directeur général de la Société Hydroélectrique de Savoie.

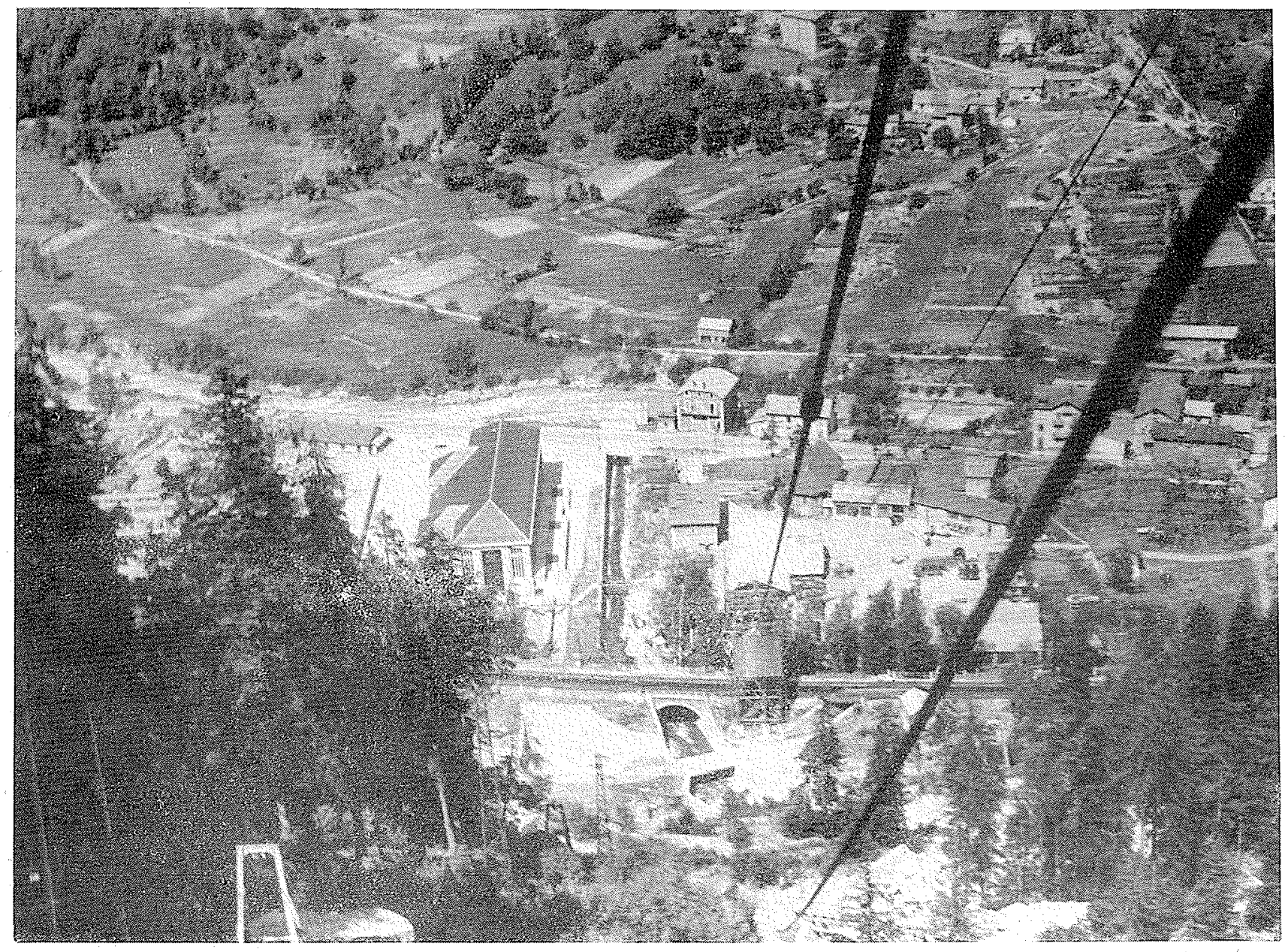

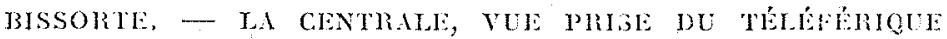


centrales dauphinoises : Chambon, SaintGuillerme, Sautel, et aux usines d"aluminium de la Durance par une ligne d'interconnexion à 150.000 volts qui enjambe le col du Galibier à 2.760 mètres d'altitude. C'est bien à notre connaissance la ligne électrique la plus ćlevée d'Eu'rope.

\section{Le bilan des installations de houille blanche en Savoie}

Tout cet ensemble d'installations d'usines hydroélectriques en Savoie représente déjà de forts belles réalisations atteignant, en 1941, une puissance de $700.000 \mathrm{k} w$ installés (1), soit près de 1.000 .000 de chevaux, classant dès lor's la Savoie au pinacle des départements francais pour la production de l'énergie électrique par la houille blanche.

L'énergie électrique qui n'esi pas employće sur place aux fabrications électrochimiques et électrométallurgiques, est transportée par les lignes à 150.000 volts : Viclaire, Albertville, Aoste, Lyon, débitant sur la grande boucle francaise à 220.000 volts : La Truyère, Lyon, Cerney, Paris, sur laquelle viennent se greffer les lignes de liaison avec les Alpes du Dauphiné, les Pyrénées, la Bretagne et l'est de la France.

\section{Génissiat 200.000 ef $400.000 \mathrm{kw}$}

S'il est vrai que la Saroie ne puisse accaparer entièrement le Rhöne, nous ne saurions manquer de signaler les magnifiques travaux en. cours à Génissiat, à cheval sur la Haute-Savoie ei l'Ain, avec barrage a travers le Rhone et centrale au pied, équipée pour $200.000 \mathrm{kw}$ (turbines de $70.000 \mathrm{kw})(2)$ dans son premier stade, puissance qui sera portée par la suite à $400.000 \mathrm{kw}$.

Il s'agit là de puissances considérables à léchelle des grandes installations du Niagara, si l'on songe que, dans son premier stade d'utilisation, le Rhône à Génissiat permettra de produire :

1.600 .000 .000 de kilowatts-heure

énergie équivalente à la production mensuells totale actuelle, hydraulique et thermique de ioutes les centrales de France.

\section{L'avenir de la houille blanche en Savoie}

La Savoie n'en restera pas là. Certes, il est hors de doute que la plus grande partie des chutes avantageuses est actuellement équipée, mais les projets qui sont à l'étude sont surtorit

(1) $700.000 \mathrm{kw}$ représente la puissance totale hydroelectrique frangaise en 1925, au moment de l'exposition de la houlle blanche de Grenoble.

(2) Ces turbines sont en construction dans les Ateliers

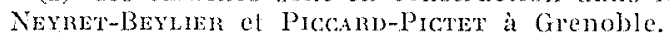

dirigés vers la création de grands barrages réservoirs, en tète des bassins des cours d'eau a régime glaciaire, barrages formant des lacs d'accumulation permettant de reporter sur l'hiver, saison des plus petits débits, les réserves accumulées pendant la saison chaude.

De récentes études, formant le dernier cri de la houille blanche en Savoie, sur:

- lisere supérieure à rignes;

- l'Are supérieur à Lanslebourg;

- Ie Doron de Pralognan a Champagny;

- l'Arve supérieure avec captation des eaux du Mont-Blane,

montrent quen utilisant des sites exceptionnels de barrages et en organisant, en fonction de ces barrages, toul l'équipement du bassin, il serait possible de réaliser une production électrique annuelle de l'ordre de 2.000 .000 .000 de $\mathrm{kwh}$, don 1.400 millions en hiver, entre le $1^{\text {er }}$ octobre et le 31 mars, et 680 millions entre le $1^{\text {ar }}$ avril et ie 30 septembre, énergie équivalente à 1.500 .000 tomnes de charbon. Ces nouvelles installations' savoyardes permettraient en hiver, saison de basses eaux de ses cours d'eau, de majorer de $70 \%$ la production d'énergie des Alpes francaises. Elles introduiraient surtout, d'apres M. Habgener, Inspecteur Général des Ponts et Chaussées, un moven de produclion extrèmement sûr de l'énergie hydraulique complètement indépendant des conditions de l'hydraulicité de l'année.

Il est remarquable de constater que les installations de Tignes et de Champagny pourraient être équipées pour une puissance de 400.000 lilowatts, soit 500.000 chevaux, représentant exactement la puissance du Rhòne à Génissiat dans sa complète utilisation.

Bref', des réalisations à l'échelle américaine qui, lorsqu'elles seront menées à bien doteront la Savoie de quelques centaines de milliers de kilowatts : $1.200 .000 \mathrm{kw}$ exactement (1), sertis à la couronne de ses forces hydroélectriques raffermissant son titre incontesté de plus grand producteur francais d'énergie de houlle blanche.

I a Savoie peul se montrer fière d'avoir donné un tel developpement à l'équipement de ses cours d'eau et apporté des techniques nouvelles comme celles que notre éminent géographe alpin Raoul BLanchard a baptisé de "technique de l'Alc » el de « technique de la Girotte». Aucun pays du monde, hormis la région des grands lacs américains, ne possède, à superficie égale, un pareil ensemble de forces hydrauliques aménagées et cela est vrai, non seulement pour la Savoie, mais pour toute la France qui, à ce point dé vue, tient toujours le troisième rang après les Etats-Unis et le Canada.

\footnotetext{
(1) 1.200 .000 kilowatts représentent la puissance totale equipée en 1939 dans les Alpes frangaise:
} 


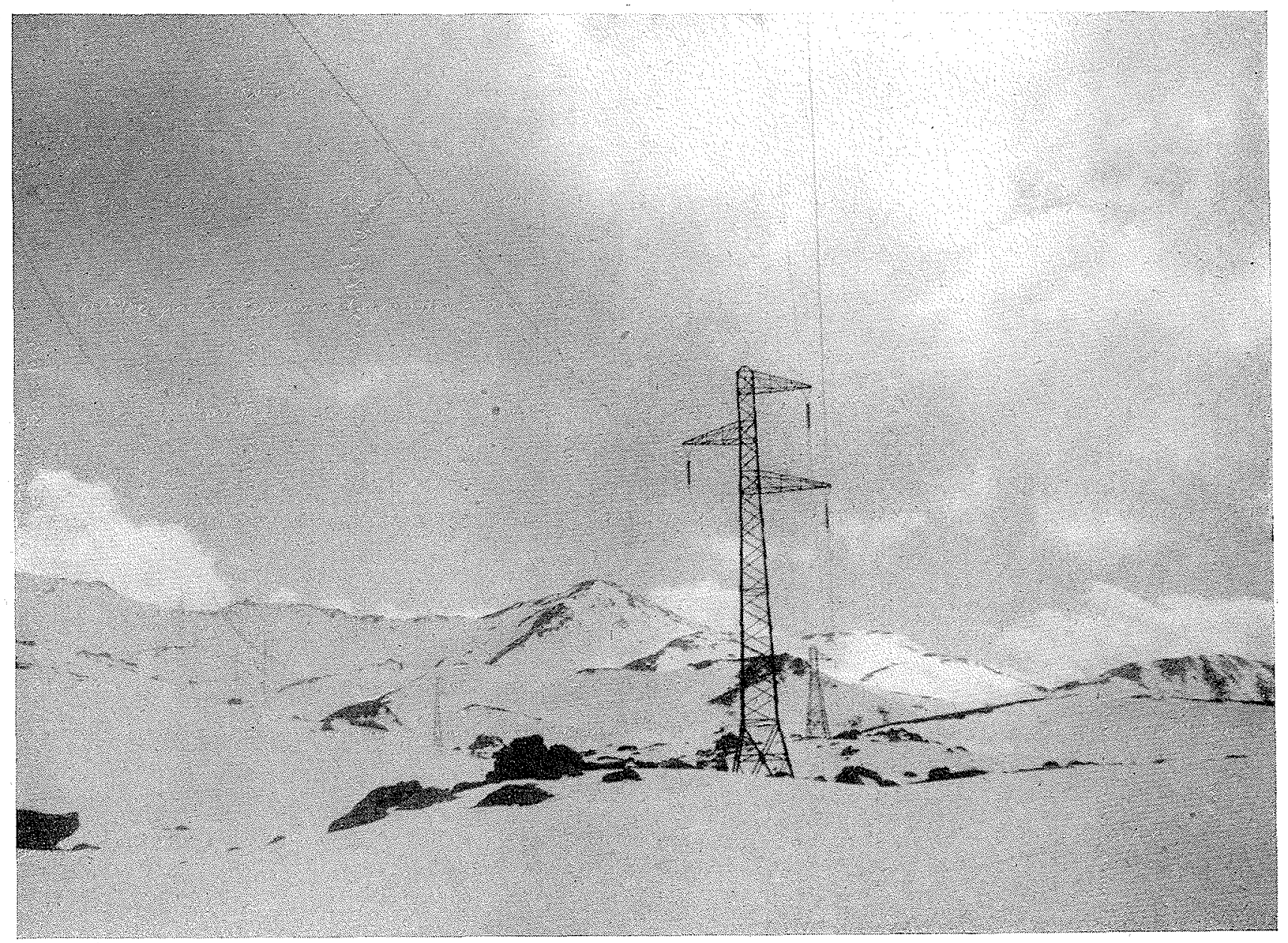

IA LIGNE 150.000 v. BISSORTE-DAUPHINÉ AU COL DU GALIBIER, $2.760 \mathrm{~m}$.

C'est une bien belle xulvire qui a été accomplie dans nos vallées savoyardes par les : Honteun, Aubry, Paul Groon, Barut, Henri G.lul, Georges Bergìs, Paul Cormin et Hippolyte Bouchayer, auxquels sont venus se joindre les grands noms d'ingénieurs, comme les : Vierhomme, Pechrner, Heronle et Miner, qui ont transformé la Maurienne en « Val d'aluminium ».

Oui, nous ne saurions trop le rappeler aujouid'hui, à une époque où certains essaient de mettre en doute la valeur de nos devanciers c'est une bien belle ouvre qu'ils ont accomplie, depuiss un demi siècle, le long des cours d'eau de notre pays.

Comme le disait déjà Raoul Blanchard, apyès l'autre guerre, ne craignons pas de le redire avec lui, car cette auve magnifique nous fait honneur à tous : elte fait homneur à ces homm-s qui n'ont pas hésité, malgré les risques, à se lancer dans ces entreprises nouvelles et l"ont fait sans timidité, voyant grand du premier coup. Elle fait honneur aux ingénieurs et aux cons- tructeurs qui ont marché, sans défaillance, en tète du progrès et qui luttent si dùrement aujourd'hui.

N'oublions pas que ce sont des Français, installés dans les Alpes du Dauphiné et de la Savoie, qui ont été les premiers à utiliser la houlle blanche, à créer de toute pièce l'outillage approprié, à résoudre les problèmes complexes d'exploitation et de fabrication.

\section{Cette houille blanche brille comme le plus beau fleuron à la couronne des montagnes de la Savoie}

Et, comme le disait si poétiquement Gabriel Hanotadx, à l'ouverture du premiér Congrès de la Houille Blanche à Grenoble en 1902 :

«Ainsi une grande révolution est accomplie, " la montagne jusque-là inactive et inféconde «va prendre part au labeur universel ; elle est

« domptée et maitrisée par son propre fils, le « montagnard. » 